\title{
DE LA COLONIZACIÓN DE LA SOCIEDAD CIVIL A LAS TENSIONES ENTRE PARTIDOS EN EL GOBIERNO Y MOVIMIENTOS SOCIALES
}

Esteban Iglesias

El propósito de este trabajo reside en revisar un aspecto central de la perspectiva de los movimientos sociales en el marco de la problemática de la acción colectiva, en particular, el planteo consistente en que las relaciones que establece el Estado con los movimientos sociales deben comprenderse en términos de cooptación, burocratización o colonización. Esto será revisado a la luz de experiencias actuales de partidos políticos que asumieron al gobierno en América Latina, sobre todo, Argentina y Brasil, con el objeto de reflexionar sobre la actualidad y fertilidad teórica de este planteo. Esta reflexión se inscribe teóricamente en el amplio campo de la sociología política y presenta resultados de proyectos de investigación en marcha.

El argumento principal de este trabajo reside en que esta hipótesis debe ser revisada en la coyuntura actual, al menos desde comienzos del tercer milenio. El estudio de los casos de Argentina y Brasil brinda indicios acerca de que los principales riesgos que acechan a los movimientos sociales no radican tanto en el carácter de su vínculo con el Estado sino, más bien, con el tipo de relación que entablan con el partido que asumió el gobierno. 
En efecto, los gobiernos encabezados por Néstor Kirchner y Cristina Fernández de Kirchner (entre 2003 y 2011) y Luiz Inácio "Lula" da Silva (entre 2003 y 2010) presentan diferencias respecto del modo en que acceden al poder político, en el tipo de liderazgo que orientan sus gobiernos y, finalmente, en el vínculo que establecen con movimientos sociales, ya sea en sus trayectorias como en la situación planteada en sus gestiones gubernamentales. Sin embargo, es posible observar que las experiencias contrastantes de los dos países conducen a resultados similares. Por su parte, en Argentina, el kirchnerismo, en tanto fuerza política, ha insistido en la construcción de "adversarios" y de esa manera se dicotomiza el espacio político. En estas batallas los movimientos sociales afines al kirchnerismo han quedado al descubierto y así se ponen en tela de juicio los principios identitarios que le dieron origen. Por otra parte, en Brasil, Lula da Silva inició un gobierno de

114 “continuidad con cambio", es decir, de no ruptura con el pasado, con un liderazgo organizado en torno al diálogo, que termina perjudicando el vínculo con uno de los principales movimientos sociales de ese país: el Movimiento Sin Tierra (MST).

El rasgo diferencial que se observa en el carácter del liderazgo de quien encabeza el gobierno constituye un elemento confirmatorio en cuanto a demostrar que el principal problema de los movimientos sociales es el tipo de relación que establecen con el partido que asumió al gobierno y no con el Estado.

\section{Las perspectivas de la acción colectiva}

En la actualidad las ciencias sociales ofrecen, en términos generales, dos perspectivas teóricas de la acción colectiva, curiosamente, las mismas que estuvieron en el origen de esta temática a mediados de la década de 1960. Entonces, el enfoque de la movilización de recursos y la perspectiva de 
los movimientos sociales compartían una misma preocupación, en especial, preguntarse quiénes eran los sujetos y las razones por las que protestaban en aquel momento. Este interrogante tenía rasgos novedosos ya que no se trataba de protestas organizadas por las tradiciones de lucha que anidaban en la clase obrera sino, más bien, de "colectivos" e identidades políticas nuevas integradas por individuos de clase media que no presentaban problemas de inserción en la economía y que inscribían sus prácticas políticas en lo que se conoció como pacifismo, ecologismo, movimiento gay, la igualdad de derechos, etc.

En este marco se originan las perspectivas de la acción colectiva, siendo su principal pregunta, se reitera, quiénes y por qué protestan ciudadanos que, aparentemente, no presentan necesidades económicas insatisfechas. Así es como, a partir de la respuesta de este interrogante, se abren las líneas de investigación teórica. Por su parte, el enfoque de la movilización de recursos considerará que la acción colectiva se pone en marcha gracias a la existencia de una serie de recursos en la sociedad siendo los más relevantes el principio de la organización y las oportunidades de lucha política ${ }^{1}$. Por otra parte, la perspectiva de los movimientos sociales planteará que lo que pone en marcha la acción colectiva es la identidad política forjada al calor de la protesta.

De los enfoques de la acción colectiva posee mayor pertinencia teórica para el análisis de los fenómenos actuales a la perspectiva de los movimientos sociales. La razón fundamental de ello radica en que permite recuperar la cultura política respondiendo de forma fehaciente el siguiente interrogante: ¿de qué modo prácticas políticas ya existentes en la sociedad se fusionan con formas de

${ }^{1}$ Desde Olson (1992), pasando por McAdam et al. (1999), hasta Tarrow (1999, 2004) y Tilly (2000) el planteo puede ser rastreado teóricamente aunque con variaciones. Para su análisis se puede consultar Iglesias (2008). 
lucha presentes y qué tipo de identidades se constituyen a partir de esta fusión?

Las definiciones de movimiento social de Touraine (1995) y de Melucci (1999) confirman este tipo de comprensión de la acción colectiva. Por su parte, Touraine (1995) señala que un movimiento social se define en torno a tres principios: el de identidad, el de oposición y el de totalidad. Por ese motivo, plantea que estos tres principios deben ser entendidos en plena interacción. En este sentido, el primero se vincularía con la definición que hace el actor de sí; el segundo, con la definición acerca de su adversario, la cual el actor tiene que hacer para tener la definición acerca de sí mismo. Finalmente, el tercero principio alude a la elaboración de un proyecto por parte del actor mediante el cual éste pondría en cuestión la historicidad ${ }^{2}$ de la sociedad. Este último punto, para Touraine, es el que reviste mayor complejidad ya 116 que no todo actor social logra articular el tercer principio, decisivo en un movimiento social.

Por otra parte, Melucci (1999, p. 46), cuya definición no difiere mucho de las dimensiones de análisis propuestas por Touraine, entiende que un movimiento social se define en torno a tres componentes: “a) basada en la solidaridad, b) que desarrolla un conflicto y c) que rompe los límites del sistema en que ocurre la acción”.

Como se observa en estas definiciones - que no son las únicas pero sí las obligatorias - se acentúa el modo en que los procesos de constitución identitaria se libran en un marco de disputa política.

\footnotetext{
${ }^{2}$ Para Touraine “[...] en el nivel del campo de la historicidad, el cambio está regido por los cambios sobrevenido en las relaciones de clase y por la capacidad de innovación de una sociedad. El cambio realiza una mutación de un tipo de sociedad a otro" (1995, p. 305).
} 


\section{La centralidad de la hipótesis de la colonización de la sociedad civil}

Esta hipótesis de trabajo se considera en un marco donde el estatus teórico de los movimientos sociales ha sido considerado inicialmente como un reemplazo fructífero para la categoría de "clase social" (Touraine, 1987) y, después, como el elemento de transformación social y democratización (Habermas, 1989; Cohen y Arato, 2000). De modo que se observa una carga normativa importante en lo que respecta a la conceptualización de los movimientos sociales y, en efecto, la pregunta que tenían que responder sus referentes teóricos era la siguiente: ¿qué razones explicaban la no constitución de movimientos sociales o, para plantearlo de otro modo, cuáles eran las dificultades por las que un sinnúmero de protestas no se convertían en movimientos sociales? En el modo en que se respondió este interrogante se abrieron dos líneas de investigación en la perspectiva de esos movimientos, una fue la delineada por Touraine y otra fue la seguida por Offe (1992), Habermas (1989), Benhabib (2006), Cohen y Arato (2000).

Por su parte, Touraine argumenta la existencia de dos factores que obstaculizan la constitución de movimientos sociales. El primero, relativo a la intervención de la clase dirigente en la construcción de la historicidad y el modo en que, a partir de esta, se desactivan formas organizativas de los sectores subalternos. Y, el segundo, se vincula a los pedidos de institucionalización por parte de los movimientos sociales, operación que para este autor redunda en la pérdida del radicalismo inicial de los movimientos ya constituidos.

En la línea de investigación que va de Offe (1992) y Habermas (1989) hasta a Cohen y Arato (2000), por otra parte, los puntos de contacto entre Estado y las diversas formas de organización de la sociedad civil aparecen 
tematizados en términos de cooptación, burocratización o colonización, por parte de las iniciativas estatales, hacia la capacidad trasformadora de los movimientos sociales. Ciertamente, las tensiones existentes en lo proceso de institucionalización política constituyen el momento más acabado de la tensión entre Estado y movimientos sociales.

En primer lugar, Offe (1992) refleja estas preocupaciones al plantear su "modelo experimental por etapas" referido a la autotransformación institucional de la política de los movimientos sociales. En este sentido, considera que a lo largo de la historia de esa institucionalización fue posible vislumbrar, en un primer momento, que el conflicto es recurrente debido a que la acción colectiva se encuentra en una instancia formativa cuyo modo de expresión privilegiado consiste en la disposición a actuar. Posteriormente, y debido a la constitución de una identidad colectiva, aparece el problema de la organización, 118 cuyo punto principal reside en cómo esta tiene que darse la tarea de regenerar de modo permanente la identidad originaria y evita las instancias burocratizadoras que eran facilitadas por la institucionalidad del sistema político.

En segundo lugar, Habermas (1989) plantea la temática de la "acción comunicativa" en el marco de los procesos de racionalización que experimentaron las sociedades modernas en Occidente. A este análisis lo lleva adelante a partir de dos términos, el de sistema ${ }^{3}$ y el de mundo de $v^{\text {vida }}{ }^{4}$. Cabe destacar que ambos términos se hallan orientados por diferentes "recursos"; así, el sistema tiene como principal recurso al "poder administrativo" mientras que el

\footnotetext{
${ }^{3}$ Alude a los mecanismos que tienden a reducir la complejidad del ambiente en el sistema social.

${ }^{4}$ Remite a la dimensión de socialización que experimentan los individuos en interacción dialógica entre sí y con las instituciones. Esencialmente se refiere a la construcción identitaria de la sociedad y a los procesos de integración que esta atraviesa.
} 
mundo de la vida a la "solidaridad". Para Habermas, durante el siglo XX, se desarrollaron procesos de "colonización" por parte del poder administrativo hacia el mundo de vida. El ejemplo más revelador se pudo vislumbrar durante la crisis de los Estados de bienestar de los países desarrollados. En esa ocasión el "ciudadano" se convirtió en un "cliente" y, también, en un "contribuyente", es decir, en un individuo que no participa ni decide de la "cosa pública" sino que se dedica a abonar impuestos.

Finalmente, el planteo de Cohen y Arato (2000), cuyo rasgo principal reside en presentar una teoría sistemática de la sociedad civil, la que es concebida como una esfera de interacción social que a diferencia de la sociedad política y la sociedad económica constituye la sede de la transformación política de la sociedad. En efecto, las tendencias a la burocratización planteadas por Habermas son convertidas por Cohen y Arato en presupuestos de la teoría, considerando que en la sociedad política y en la sociedad económica los actores participan de forma directa en el poder del Estado y en el de la producción económica, “[...] a los cuales procuran controlar y manejar" (Cohen y Arato, 2000, p. 9). En cambio, la lógica de la acción política se basa en el recurso de la solidaridad y el "entendimiento", a partir de los cuales los individuos deliberan en la esfera pública. En la esfera de la sociedad civil no hay un control directo sobre el poder ni sobre las decisiones que se toman sino, más bien, lo que se intenta es "influenciar" sobre la sociedad política con la pretensión de que las iniciativas estatales asuman un carácter legítimo. Por último, se destaca que la sociedad civil estaría compuesta por "[...] la esfera íntima (en especial la fami-

\footnotetext{
${ }^{5}$ En este sentido, señalan que "[...] con la generación de influencia mediante la actividad de las asociaciones democráticas y la discusión no restringida en la esfera pública cultural. Tal papel político es inevitablemente difuso e ineficaz" (Cohen y Arato, 2000, p. 9).
} 
lia), la esfera de las asociaciones (en especial las asociaciones voluntarias), los movimientos sociales y las formas de comunicación pública" (Cohen y Arato, 2000, p. 8).

En todos estos intentos por teorizar sobre movimientos sociales y diversas formas organizativas de la sociedad civil se entiende que los procesos de democratización por parte del Estado han fracasado históricamente. Y, el costado ligero de la transformación política es el de la esfera de la sociedad civil. En definitiva, las iniciativas estatales son consideradas negativamente $y$, por ende, todo punto de contacto de la sociedad civil con éstas se encuentra a colonización. Obviamente, esto tiene variaciones y acentos, sin embargo, las soluciones teóricas y la dimensión normativa de las propuestas teóricas se encuentran por el ámbito de la sociedad civil y no por el Estado ${ }^{6}$.

\section{Mención a los aspectos metodológicos}

120 Como se señaló, este trabajo se ha propuesto reflexionar sobre un nudo problemático al interior de la perspectiva de los movimientos sociales. También se indicó que se exponen resultados de investigaciones $\operatorname{previas}^{7}$, con lo cual se justificará los elementos que se compararon en los estudios de caso y se mencionará el modo en que se realizó la recolección de la información.

Con respecto a los casos bajo análisis cabe mencionar que el criterio de selección de partidos políticos y movimientos sociales fue su importancia política en la actualidad y su grado de representatividad. En el caso de Brasil optamos por el Partido de los Trabajadores (PT), la Central

\footnotetext{
${ }^{6}$ La propuesta de "democracia deliberativa" en la esfera pública de Habermas (2010) es una muestra cabal de esto.

${ }^{7}$ En calidad de investigador del proyecto "Viraje ideológico y emergencia de formas políticas alternativas. Partidos y movimientos de la nueva izquierda en Sudamérica" dirigido por el Doctor Arturo Fernández y radicado en Conicet y en la codirección del proyecto de investigación "Gobierno, protesta social y sectores populares” radicado en Universidad Nacional de Rosario.
} 
Única de los Trabajadores (CUT) y el MST mientras que en Argentina por el Partido Justicialista (PJ), la Confederación General del Trabajo (CGT) y las organizaciones piqueteras. Así, se seleccionaron dos expresiones del movimiento sindical y expresiones de movimientos social. Se entiende que existe un rasgo en común entre el MST y los "piqueteros": la desposesión que hundió raíces en su constitución identitaria. En este sentido, el MST se constituyó peticionando por la falta de "tierras" mientras que los piqueteros por la ausencia de "trabajo". Se entiende que existe pertinencia metodológica en el conjunto de elementos a comparar en las experiencias de Argentina y Brasil.

La técnica principal de recolección de información ha sido la "entrevista en profundidad" realizada a informantes claves y, en menor medida, se ha acudido a la revisión de noticias periodísticas.

La entrevista en profundidad tuvo un carácter no estructurado permitiendo distinguir etapas y temporalidades por las que atravesó el vínculo entre Estado, partidos políticos y movimientos sociales. El guión de entrevista contempló temas y sub-temas que remitieron a las causas y especificidades sobre las que se construyó el vínculo entre iniciativas estatales, partidos y movimientos sociales. La información brindada por los informantes claves permitió distinguir tipos de relación entre Estado y movimientos sociales así como entre partidos en el gobierno y movimientos sociales. Esto proporcionó información relevante para determinar el carácter de las iniciativas estatales, las prácticas políticas de los partidos políticas y su incidencia en el carácter cambiante del accionar colectivo de los movimientos sociales.

Los informantes claves fueron seleccionados por ser referentes políticos de máxima responsabilidad en las organizaciones políticas, por conocer su historia, por participar en el proceso de toma de decisiones y por brindar conocimientos específicos acerca de los criterios que motivan las 
acciones colectivas. Los encuentros con los informantes claves, en la ciudad de San Pablo, Brasil, tuvieron lugar en 2010 y 2011, pudiéndose registrar doce en total. En Argentina, en la ciudad de Buenos Aires y Rosario se mantuvieron encuentros en los años 2005, 2010 y 2011, pudiéndose registrar quince. La duración de los encuentros fue de 60 a 90 minutos.

Finalmente, el seguimiento periodístico cumplió la función, por un lado, de brindar elementos para caracterizar el contexto político y, por otro lado, de permitir contrastar y mensurar la información bridada por los informantes claves. Los medios de comunicación gráficos consultados fueron para Argentina periódicos de alcance nacional - Clarín y La Nación - como de tirada local - La Capital - y, para Brasil, Folha de São Paulo.

\section{Puesta a prueba y revisión de hipótesis de trabajo}

El problema relativo a las relaciones de cooptación, buro122 cratización o colonización del Estado o "esfera gubernamental" hacia la sociedad civil y los movimiento sociales, ha tenido buena recepción en América Latina. También, cabe destacar que es una temática que ha resurgido desde el comienzo del tercer milenio, sobre todo, por el acceso al poder político de partidos políticos que se constituyeron en vinculación con movimientos sociales - como fue el caso del PT - o que luego de acceder al gobierno recibieron su apoyo - como fue el caso del kirchnerismo.

En este sentido, podemos mencionar algunos trabajos que han replicado esta idea: Bobes $(2010)^{8}$, Druck $(2006)^{9}$,

\footnotetext{
${ }^{8}$ En lo que respecta a los vínculos establecidos entre Estado y sociedad se entiende que "[...] la sociedad civil supone el establecimiento de relaciones horizontales (que desafían y resisten la verticalidad de las relaciones Estado-Sociedad) [...]" (Boves, 2010, p. 44).

${ }^{9}$ En referencia al vínculo gobierno de Lula y movimientos sociales la autora plantea los riesgos sobre la re-organización de los movimientos sociales desde el Estado hacia la sociedad civil (Druck, 2006).
} 
Gohn $(2009)^{10}$, Levitsky (2004) ${ }^{11}$, Massetti (2009) ${ }^{12}$, Svampa $(2005)^{13}$, Zuazo $(2010)^{14}$, etc.

El análisis del vínculo entre partidos en el gobierno y movimientos sociales no ha sido desatendido, sin embargo, en el marco de la perspectiva de los movimientos sociales se ha enfatizado la interpretación en su vinculación con el Estado. Esta hipótesis, se considera, debe ser revisada y matizada. Tal vez el elemento que puede oficiar en el análisis es el tipo de liderazgo que se ejerce desde el partido que conduce el gobierno.

Esta problemática no es actual, ciertamente. De todas maneras, la personalización de la política ha venido de la mano de transformaciones que tuvieron lugar en el plano de la representación política. Tal como plantea Manin (1998) los cambios implicaron modificaciones en el modo en que se constituyen las identidades políticas. En esta ocasión el programa político de los partidos

10 "Uma das hipóteses sobre a fragilidade dos movimentos sociais no Brasil, neste novo milênio, é que eles perderam força política como agentes autônomos porque se transformaram em meios de institucionalização de práticas sociais organizadas de cima para baixo, práticas que são formas de controle e regulação da população. Sabe-se que a conquista do poder político por setores que anteriormente estavam na oposição, em importantes aparelhos do Estado, levou a ampliação de políticas sociais voltadas para os excluídos, para criar redes de proteção aos chamados bolsões humanos de vulnerabilidade social. Mas isso não significa que houve fortalecimento das organizações populares. Ao contrario, muitas delas enfraqueceram-se. Seus líderes foram cooptados pelos aparelhos estatais e suas políticas compensatórias" (Gohn, 2009, p. 60).

${ }^{11}$ Desarrolla el modo en que el PJ desde la restauración democrática hasta 1999 se transformó en un partido clientelista modificando así su vínculo con los sectores populares.

${ }^{12}$ Desarrolla detalladamente cómo se dio esta incorporación al gobierno de la ciudad de Buenos Aires, presidido en ese entonces por Jorge Telerman. El autor concluye que este intento de incorporación al Estado estuvo fuertemente signado por un contexto de electoral de renovación de jefe político en la ciudad de Buenos Aires, lo que culminó en una utilización de los dirigentes de los movimientos sociales. ${ }^{13}$ Para la autora la participación de organizaciones piqueteras en el gobierno ha derivado en cooptación política.

${ }^{14}$ En referencia a Bolivia plantea que " $[\ldots]$ lo que se observa es una domesticación de las organizaciones sociales a partir de una estrategia de fragmentación y apropiación de la iniciativa política y organizativa” (Zuazo, 2010, p. 135). 
dejó de ser un elemento diferenciador de las identidades, quedando relegado y sustituido por la "imagen del candidato" reproducida en una nueva escena, que no era la plaza pública, sino los medios audio-visuales de comunicación masiva. Así, para Manin estas transformaciones en la representación política implicaron cambios en el tipo de democracia, constituyéndose una "democracia de audiencia" en la que el ciudadano se comporta por impulsos de acuerdo a la oferta electoral.

Del planteo de Manin es posible rescatar la cuestión de la re-personalización de la política en una nueva arena, la de los medios masivos de comunicación. Para el análisis, a este elemento hay que sumarle que las iniciativas estatales de los gobierno en América Latina ya no son más de corte neo-conservador. Más bien, intentan reparar situaciones sociales que experimentó la ciudadanía durante la década anterior: desempleo, sub-ocupa-

124 ción, reducción de salarios, problemas de salud, vivienda y protección social.

En este marco de transformaciones en la representación política, en el comportamiento político de la sociedad y en el carácter de las iniciativas estatales lo que ingresa como nuevo problema para los movimientos sociales es su vínculo con los partidos políticos en el gobierno y no tanto las relaciones con el Estado. La piedra de toque de esto es el tipo de liderazgo de quien conduce el gobierno. Por ello, a continuación relatamos dos modos de ejercer el liderazgo, uno basado en el "diálogo" como es el caso de Brasil y otro organizado en torno a la "construcción de adversarios" como es el caso de Argentina.

\section{Argentina, el liderazgo de confrontación}

¿Cómo se constituyó el kirchnerismo en términos identitarios? ¿Qué decisiones relevantes se tomaron y que, posteriormente, formaron parte de la constitución política 
del kirchnerismo? Néstor Kirchner al asumir el gobierno nacional heredó una situación política signada por la fragmentación de partidos en general, división del peronismo en particular, división sindical ${ }^{15}$ y organizaciones piqueteras instaladas en el escenario político nacional ${ }^{16}$.

Así, cabe destacar que el kirchnerismo, en tanto fuerza política, definió su campo identitario con un discurso de oposición a las políticas neo-conservadoras. En términos de práctica política el espacio de acción política se forjó gracias a la construcción de diversos "adversarios". Biglieri (2008) desarrolla el modo en que Kirchner en los primeros treinta días de gobierno había designado un conjunto de adversarios políticos, siendo los principales las fuerzas armadas y militares en general, la "mayoría automática" de la Corte Suprema de Justicia de la Nación, el Fondo Monetario Internacional, las empresas multinacionales, etc. Lo cierto fue que lejos de ser una situación coyuntural se convirtió en un verdadero estilo político que hunde raíces en la dimensión identitaria de la fuerza política que encabeza el gobierno nacional.

Con respecto al vínculo con los representantes del movimiento obrero y los de los movimientos sociales es posible destacar varios aspectos. En primer lugar, Néstor Kirchner el primer año de su mandato convocó al diálogo político a las organizaciones piqueteras. Este diálogo en vez de transcurrir en espacios institucionales se convirtió en un intercambio político en que se les ofreció la gestión de $\operatorname{programas}^{17} \mathrm{y}$

\footnotetext{
${ }^{15}$ Existían tres nucleamientos sindicales: la Central de Trabajadores Argentinos, la CGT rebelde y la CGT oficial.

${ }^{16}$ También recibió una estructura social transformada, que había experimentado problemas de integración social y un mercado laboral altamente fragmentado con altas tasas de desocupación y sub-ocupación y, finalmente, con una economía informal de envergadura inédita en el país.

${ }^{17}$ Según los periódicos Clarín y La Nación de 8 de julio de 2003, los programas son "El hambre más urgente", "Manos a la obra", y el "Plan arraigo". El primero es un programa de ayuda alimentaria, el segundo de construcción de viviendas comunitarias y el tercer de emprendimientos productivos.
} 
cargos políticos ${ }^{18}$ a las organizaciones a cambio del apoyo a su gobierno. Este ofrecimiento fue aceptado por Federación de Tierra y Vivienda (FTV) y por Barrios de Pie mientras que la Corriente Clasista y Combativa (CCC) y el Movimientos Independiente de Jubilados y Pensionados no aceptaron, restringiendo su vinculación a la firma de convenios que efectivizarían la realización de estos emprendimientos productivos ${ }^{19}$. Con esto el gobierno nacional logró, fragmentariamente, el apoyo político de parte de las organizaciones, inclusive, alentó su constitución desde el Ministerio de Desarrollo Social. Éste fue el caso del Movimiento Evita, que gestiona el programa de Promotores Territoriales para el Cambio Social.

El vínculo con organizaciones piqueteras se convirtió en una suerte de "premios y castigos" que se otorgaban desde el gobierno nacional. Sin embargo, la dimensión identitaria se puso en juego cuando el liderazgo presi126 dencial se disponía a la construcción de adversarios políticos, ciertamente, de envergadura solicitando que las organizaciones salieran en su apoyo y defensa. Esto se activó muy rápidamente, desde los inicios del kirchnerismo en el poder. Así, las empresas multinacionales, en particular, las expendedoras de gasoil y nafta fueron señaladas por el entonces presidente como los "enemigos del pueblo" ya que decidieron de modo unilateral

\footnotetext{
${ }^{18} \mathrm{El}$ ofrecimiento de cargos tampoco tardó en llegar siendo el más resonante fue, sin dudas, el caso de Luis D`Elía, de la FTV, en la Subsecretaria de Tierra y Vivienda a nivel nacional durante el año 2005. Otros nombramientos, no tan resonantes, fueron: el de Jorge Ceballos del Movimiento Barrios de Pie, nombrado director de asistencia comunitaria del Ministerio de Desarrollo Social en el año 2004; el de Emilio Pérsico, titular del Movimiento Evita, nombrado subsecretario de políticas públicas del gobierno de la provincia de Buenos Aires en noviembre de 2005; menos conocido fue el nombramiento, en el año 2006, de "Lito" Borello y Juan Pablo Cusa, designado por el Movimiento Evita, en el Ministerio de Derechos Humanos y Sociales del gobierno de la ciudad de Buenos Aires etc. (Massetti, 2009).

${ }^{19}$ Para los referentes políticos de la CCC este ofrecimiento era concebido como una forma de "venderle el alma al diablo".
} 
un aumento de precios en la venta de combustibles al público. En esa ocasión la FTV, encabezada por D’Elía, bloqueó, con el objeto de que no funcionen, diversas estaciones de servicio de combustibles al público. Esto tuvo gran repercusión en los medios masivos de comunicación y, también, efectividad ya que las empresas Shell y Esso decidieron bajar el precio del combustible.

Este tipo de intercambio y lógica de acción política se pudo observar cuando se desató la crisis con las entidades del agro en 2008, apenas haber asumido Cristina Fernández de Kirchner. El conflicto se originó por el tercer aumento al impuesto de las retenciones móviles de la exportación de cereales y oleaginosas, particularmente, trigo, maíz y soja. Esta acción de gobierno derivó en un hecho histórico, que pequeños productores del campo constituyan una alianza, formalizada en la denominada "mesa de enlace", con la oligarquía agropecuaria. Luego de varios meses de disputa política, donde el gobierno intentó sin éxito dicotomizar el espacio político entre lo "nacional y popular" expresado por la autoridad presidencial y los intereses de la "oligarquía del campo". Entonces, ¿qué papel jugaron las organizaciones piqueteras en este conflicto? No sólo apoyaron las decisiones del oficialismo sino que Luis D`Elía intentó desalojar del espacio público, a golpes de puño, a un manifestante que apoyaba a las organizaciones agrarias. Esto es un claro ejemplo de cómo el vínculo con el partido en el gobierno asumió una relevancia poco mensurada en identidades políticas relativamente jóvenes y que carecen de la fortaleza de otras.

La relación con el movimiento sindical revestía mayor complejidad que la de los movimientos sociales surgidos en la década de 1990 en Argentina. Como se planteó, se asistía a una situación de fragmentación sindical, la que se intentó recomponer desde el oficialismo. En efecto, durante 2004 y 2005 el gobierno nacional logró la unificación de la CGT, 
bajo la conducción del camionero Hugo Moyano ${ }^{20}$ ¿ ¿Qué características asumió la CGT liderada por Hugo Moyano en los sucesivos gobiernos kirchneristas?

Cabe destacar que el intercambio político entre kirchnerismo y sindicalismo peronista revestía de complejidad debido al poder de las fuerzas políticas en juego. Ciertamente, eran las fuerzas que mayor poder de movilización ciudadana presentaron en este período. En cuanto a su relación, en términos generales, obedeció a una estructura de intercambio basada en que la CGT apoyaba al gobierno y, como contrapartida, se permitía una estrategia política de penetración de las organizaciones gremiales dirigida al interior del partido peronista. La importancia de este hecho era capital: desandar la desarticulación histórica entre partido peronista y sindicalismo peronista iniciada a mediados de la década de 1980.

Fortalecido el kirchnerismo y el liderazgo de Hugo 128 Moyano en la CGT, luego de 2005, y, en un contexto de crecimiento económico y de recuperación del empleo, el papel de la CGT se viabilizó en el plano institucional, participando en el Consejo del Salario Mínimo y en el de las Convenciones Colectivas de Trabajo. En esta instancia institucional la negociación colectiva efectuada por el sindicato de camioneros oficiaba de pauta de aumento salarial y de "techo" para trabajadores nucleados en otras asociaciones gremiales. Según el estudio de Etchemendy y Colliers (2008) se demuestra que las huelgas registradas por reclamo salarial y mejora en las condiciones de trabajo se daban en el momento en que los representantes sindicales participaban de la negociación paritaria. Con lo cual se observaba que era una forma de presión política y activada coyuntural-

\footnotetext{
${ }^{20}$ En 1994 Moyano, junto a Juan Manuel Palacios, formó el Movimiento de Trabajadores Argentinos. Era un nucleamiento sindical de tradición peronista que no apoyaba políticas neo-conservadoras, con lo cual no era pro-gobierno nacional. Éste, desde 1999, se denominó CGT-rebelde.
} 
mente, para reforzar los reclamos. Por casualidad, las protestas finalizaban cuando se cerraba el acuerdo paritario. Esta mecánica política se mantuvo desde 2005 hasta 201121.

Los apoyos políticos por parte del gremialismo peronista hacia el kirchnerismo se hacían visibles en los actos políticos y, también, en los conflictos en los que se embarcaba el partido en el gobierno. Como sucedió con el movimiento piquetero, el conflicto con las entidades del agro en 2008, fue decisivo porque para Moyano este apoyo le ganó la enemistad de quien conduce las 62 Organizaciones, el brazo sindical que actúa en el partido peronista.

Curiosamente, tardaba en concretarse la penetración sindical hacia el interior del partido peronista. En este sentido, el proyecto político de Hugo Moyano quedó inconcluso. Logró diversas conquistas gremiales, sin embargo, no avanzó en su desarrollo político-partidario. Hoy es un político marginal al proyecto político que encabeza Cristina Fernández de Kirchner. En la coyuntura electoral de 2011, momento de la re-elección de la actual presidenta los alineamientos políticos se modificaron. Ese momento estuvo signado por la muerte de Néstor Kirchner, y la actual presidenta decide constituir una fuerza política propia en la que se retacea el apoyo del sindicalismo peronista y se incorporan jóvenes militantes peronistas. De estas agrupaciones la de mayor relevancia es la fundada por su hijo, Máximo Kirchner, denominada La Cámpora. El legado político de Néstor Kirchner es ahora interpretado por la persona que se halla mejor autorizada para hacerlo, Cristina Fernández de Kirchner. Así, los elegidos

\footnotetext{
${ }^{21}$ Según el periódico La Capital, Néstor Kirchner mantuvo una reunión con Hugo Moyano el 20 de abril de 2007 con el objeto de colocar un tope de 16,5\% de aumento de salarios a federaciones y uniones de envergadura. Así, días más tarde nucleamientos sindicales poderosos como Unión Personal Civil de la Nación (UPCN) y la Unión Obrera de la Construcción (UOCRA) cerraban los acuerdos paritarios bajo esta consigna.
} 
para defender su proyecto político han sido los "jóvenes" y políticos de confianza en el cual se relega al líder de la CGT, Hugo Moyano.

\section{Brasil: PT, CUT'22 y MST}

El PT, liderado por Lula da Silva, asume el gobierno con el apoyo de diversos movimientos sociales. Con algunos tenía mayor proximidad - CUT, Unión Nacional de Estudiantes (UNE), Marcha Mundial de las Mujeres, etc. - y con otros una menor - MST, pastorales católicas, etc. En esta oportunidad seleccionaremos para el análisis el vínculo del PT con la CUT y el MST.

Al igual que en Argentina, se entiende que el tipo de liderazgo de quien conduce el gobierno, basado en el diálogo, constituyó un elemento importante en las políticas públicas implementadas y en las acciones colectivas que organizaron las diferentes expresiones del movimiento sin130 dical y social, en particular, la $\mathrm{CUT}^{23}$ y el MST. Lo cierto fue que la orientación dialógica del entonces presidente

\footnotetext{
${ }^{22}$ La Central Única de los Trabajadores (CUT) se funda en 1980, en São Bernardo Do Campo, San Pablo, dos años después de la CONCLAT, el primer Congreso Nacional de la Clase Trabajadora en Brasil, del que en 2011 se hicieron innumerables celebraciones en conmemoración de los 20 años. Esta organización ha sido constituida por diversos grupos sindicales que a finales de la década de 1970 realizaron una serie de huelgas que paralizaron la región del ABC paulista y las demás ciudades industriales cercanas a San Pablo. A fines de los setenta, un conjunto de sindicalistas realizó una serie de huelgas en las empresas petroleras, automotrices y metalúrgicas. Sin lugar a dudas, esto puso en tela de juicio uno de los pilares de la dictadura militar: la prohibición de realizar huelgas.

${ }^{23}$ El vínculo entre PT y CUT ha sido extensamente explorado. Mencionamos bibliografía básica sobre la temática y los diferentes enfoques teóricos. Así encontramos estudios que abordan la génesis compartida - Meneguello (1989) y Keck (1991); las trayectorias y estrategias sindicales y políticas llevadas a cabo por cada uno durante la década de 1980 para resaltar el cambio con la propuesta original; la burocratización de la CUT y el PT, la morfología singular que adquiere el entendimiento entre ambas organizaciones en la década de 1980 - Soares (2005), Oliveira (2003), Riethof (2004); la vinculación dirigencial e ideológica entre cutistas y petistas - Rodrigues $(2002,2004)$; hasta los que observan la relación entre ambas organizaciones una vez que Lula da Silva obtuvo la presidencia en 2002 - Galvão (2004), Radermacher y Melleiro (2007), entre los principales.
} 
de Brasil tenía un costado institucional, relativo a las condiciones de asunción al gobierno nacional. En efecto, el PT asume el gobierno liderando una coalición política, cuya novedad residía en aliarse con el Partido Liberal (PL), liderado por el empresario José Alencar. La historia electoral del PT muestra una estrategia "coalicional"24. Sin embargo, llamaba la atención que un partido que hunde raíces identitarias en la representación de los trabajadores (Jakobson, 2011) efectúe una alianza con una organización representada por un empresario y con una orientación político-ideológica alojada en un sector opuesto del espectro político.

El diálogo político fue iniciado por el gobierno de Lula luego de la "reforma del sistema previsional de los empleados públicos", tentativa que fue presentada de forma unilateral y sin consulta previa a los nucleamientos sindicales en que se apoyaba el gobierno. Esta iniciativa implicaba una rigidez en los criterios de jubilación públicos así como un aumento en la edad jubilatoria (Radermacher y Melleiro, 2007). Este modo de asumir el liderazgo tuvo un costo político, la formación de Coordinación Nacional de Luchas (Conlutas), en 2004, que constituyó un desprendimiento político de la CUT.

La vuelta al diálogo político se tuvo lugar cuando organizó las Conferencias o Foros nacionales, en los que el gobierno nacional mediaba entre representantes de sociedad civil y el empresariado. Uno de los más relevantes fue el Foro Nacional del Trabajo, que comenzó en 2004. Para los dirigentes sindicales fue un gran desafío político: "Os empresários todos bonitinhos, sentadinhos, empresários da

\footnotetext{
${ }^{24}$ En 1989, en su primera candidatura presidencial, Lula optó por formar el Frente Brasil Popular, del que participaron el Partido Comunista do Brasil (PCdoB) y el Partido Socialista Brasileiro (PSB). A lo largo del tiempo este esquema de alianzas se mantuvo y amplió, por ejemplo al Partido Democrático Trabalhista (PDT), siendo lo distintivo, en 2002, la inclusión a su coalición electoral de sectores políticos de la derecha de Brasil en la fórmula presidencial.
} 
indústria, do comercio, da agricultura, das finanças, como se não tivessem problemas entre eles, mas todos se comportando unitariamente e nós brigando na mesa"25. Lo más relevante de esta iniciativa política fue el reconocimiento por parte del Estado de las centrales sindicales más importantes en cantidad de afiliados y afincamiento territorial: CUT, Fuerza Sindical (FS), Unión General de los Trabajadores (UGT), Central de los Trabajadores y Trabajadoras de Brasil (CTB), Confederación Central de los Trabajadores (CGT) y Nueva Central Sindical (NCS) ${ }^{26}$.

La cuestión de alojarse en un espacio político apropiado o productivo para la CUT fue problemática en el primer período de gobierno de Lula. El margen de maniobra político de la CUT remitía, en parte, con la situación económica, con el escaso crecimiento del producto bruto interno que mostraba el país y, en buena medida, con los problemas relativos a la representación política. Así, al interior del nucleamiento 132 sindical se planteaba la creencia que "Então nós elegemos o Lula, o Lula vai pegar a caneta e vai escrever: artigo primeiro: implantação do socialismo; artigo segundo: revogam-se as disposições em contrário" 27 .

Esta situación pudo ser revertida en el segundo mandato de Lula, luego de haber transitado con éxito la acusación de corrupción conocida como "mensalão"28. Así, en el segundo período de gobierno el margen de maniobra de la CUT se amplió, pudiendo peticionar al gobierno nacional e inclusive haciendo efectiva la actualización y el aumento

\footnotetext{
${ }^{25}$ Entrevista en profundidad a Arthur Henrique, presidente de la CUT.

${ }^{26}$ Conlutas no reúne los requisitos para el reconocimiento estatal.

${ }^{27}$ Entrevista en profundidad a Artur Henrique, presidente de la CUT.

${ }^{28}$ En esa ocasión Lula recibió un amplio apoyo de los movimientos sociales, los que se encontraban aglutinados en la Coordinadora de Movimientos Sociales (CMS), encabezada por Antonio Spis, dirigente político de la CUT. Hicieron manifestaciones, declaraciones públicas - Carta al Pueblo Brasilero, publicada el 22 de junio de 2005 - y, finalmente, fueron recibidos por Lula en la Casa de Gobierno, Brasilia.
} 
del salario mínimo y, además, la reducción de la jornada laboral de 44 a 42 horas semanales.

Esta estrategia política de Lula de "huir de los dos extremos" tuvo sus costos: el vínculo con el MST se deterioró notablemente. Esto tiene una explicación política concreta y remite a las políticas públicas. El MST fue convocado por el gobierno nacional un sinnúmero de veces, siendo la más importante para la elaboración del Plan Nacional de Reforma Agraria. Lo pactado entre las partes consistía en la recuperación de 800 mil hectáreas de tierras en el lapso de ocho años, es decir, dos periodos gubernamentales del PT. Sin embargo, ello ni siquiera ha comenzado. Oliveira (2009) demuestra el escaso alcance que tuvo la reforma agraria en Brasil. Este autor discrimina entre familias que durante la presidencia de Lula ha re-asentado, reordenado, regularizado y, estrictamente, las que fueron parte de la reforma agraria. En este último caso, hasta el 2008, se observa que el gobierno nacional ha asentado 183.308 familias nuevas. Esta cifra se encuentra muy lejos de los 800 mil asentamientos que fueron acordados entre el gobierno de Lula y las diferentes expresiones del movimiento campesino en la política de la reforma agraria. Y, el revés de esta política eran los beneficios que recibía un sector del empresariado a cargo del agro-negocio. Las diferencias políticas con el PT se orientan en parte al tipo de liderazgo, así para los dirigentes del MST “[...] no se puede agradar a griegos y troyanos $[\ldots]^{\prime 29}$.

Las tensiones políticas han llevado al MST a dejar de participar de la CMS y está apostando a la Asamblea Popular, un nuevo espacio político que aglutina los mismos movimientos sociales que la CMS pero con características políticas diferentes, de confrontación con el gobierno nacional. Por otra parte, se observa un acercamiento

\footnotetext{
${ }^{29}$ Entrevista en profundidad a dirigente del MST.
} 
inédito del MST a Fuerza Sindical, un nucleamiento con el que históricamente ha presentado diferencias políticas e identitarias. Sin embargo, la relación de tensión con el partido en el gobierno generó un acercamiento ${ }^{30}$ entre estas organizaciones políticas.

La hipótesis consistente en que lo que se activa desde el Estado hacia los movimientos sociales se traduce en instancias de cooptación, burocratización o colonización ha sido fértil teóricamente para interpretar la crisis de los Estados de Bienestar en Europa a comienzos de los años de 1960. Esta situación pudo prolongarse al período posterior gracias a que las iniciativas estatales asumían un carácter neoconservador.

Esto, se plantea, debiera ser revisado, al menos en Amé134 rica Latina donde la situación política se modificó y donde la sociedad experimentó profundas transformaciones en su estructura social y en su comportamiento político. Lo cierto es que desde comienzos del tercer milenio las iniciativas estatales han tendido a reparar situaciones sociales precedentes. En lo que respecta al vínculo entre Estado y movimientos sociales se observa que el problema se trasladó de un lugar a otro, registrándose como tensión principal el vínculo con el partido político que accedió al poder político.

El análisis de nucleamientos sindicales y movimientos sociales en Argentina y Brasil constituye una muestra cabal de este problema. A pesar de las diferencias políticas y en el tipo de liderazgo las tensiones registradas son similares. En Argentina se observa un liderazgo que construye permanentemente adversarios y que se embarca en batallas de enverga-

\footnotetext{
${ }^{30}$ En la celebración del 1 de Mayo en 2011 pudo observarse la presencia de muchos movimientos sociales entre los que participa el MST, Fuerza Sindical y no participa la CUT.
} 
dura, lo que pone en riesgo la construcción identitaria de los movimientos sociales. En cambio, en Brasil se observa que un liderazgo basado en el diálogo puede llevar a perder el apoyo de movimientos sociales, en particular, del MST, además de los desprendimientos que experimentó la CUT a lo largo de los dos períodos gubernamentales de Lula da Silva ${ }^{31}$.

Esta situación constituye un desafío para los movimientos sociales: no apoyar al partido en el gobierno implica ceder espacios a expresiones y fuerzas políticas de otras orientaciones ideológicas mientras que apoyarlos los coloca en el riesgo de minar la base de apoyo sobre la que se erigieron.

\section{Esteban Iglesias}

é pesquisador-assistente no Conicet e professor de ciência política da Flacso e da Universidade Nacional de Rosario.

\section{Referências bibliográficas}

BENHABIB, S. 2006. Las reivindicaciones de la cultura: igualdad y diversidad en la era global. Buenos Aires: Katz.

BIGLIERI, P. 2008. "Nueva conceptualización sobre el populismo en América Latina”. In: FERNÁNDEZ, A.; LESGART, C. (orgs.). La democracia en América latina: partidos políticos y movimientos sociales. Rosario: Homo Sapiens.

BOBES, V. C. 2010. "De la revolución a la movilización: confluencias de la sociedad civil y la democracia en América Latina”. Nueva Sociedad, n. 227, pp.32-50.

COHEN, J.; ARATO, A. 2000. Sociedad civil y teoría politica. México, D. F.: Fondo de Cultura Económica.

DRUCK, M. G. 2006. "Os sindicatos, os movimentos sociais e o governo de Lula: cooptação e resistência. Observatorio Social de América Latina, ano 6, n.19, pp.335-40.

ETCHEMENDY, S.; COLLIERS, R. 2008. "Golpeados pero de pie: surgimiento sindical y neocorporativismo segmentado en Argentina (20032007)". Postdata, n.13, pp.141-92.

\footnotetext{
${ }^{31}$ En referencia a la constitución de Conlutas en 2004 y CTB, desafiliada de la CUT en 2007.
} 
GALVÃO, A. 2004. "La CUT en la encrucijada: impactos del neoliberalismo sobre el movimiento sindical combativo”. Revista Venezolana de Economía y Ciencias Sociales, v.10, n.1, pp.219-39.

GOHN, N. G. 2009. Novas teorias dos movimentos sociais. São Paulo: Loyola. HABERMAS, J. 1989. Teoría de la acción comunicativa. Buenos Aires: Taurus. 2010. Facticidad y valide: sobre el derecho y el Estado democrático de derecho en términos de la teoría del discurso. Madrid: Trotta.

IGLESIAS, E. 2008. "Política y protesta: visiones comparadas de la acción colectiva”. In: FERNÁNDEZ, A.; LESGART, C. (orgs.). La democracia en América latina: partidos políticos y movimientos sociales. Rosario: Homo Sapiens.

JAKOBSON, K. A. 2011. "El PT en el gobierno o el desafío de mantener las convicciones". Nueva Sociedad, n.234, pp.60-73.

KECK, M. E. 1991. PT: a lógica da diferença - o Partido dos Trabalhadores na construção da democracia brasileira. São Paulo: Ática.

LEVITSKY, S. 2004. "Del sindicalismo al clientelismo: la transformación de los vínculos partido-sindicatos en el peronismo, 1983-1999”. Desarrollo Económico, v.44, n.173, pp.3-32.

MANIN, B. 1998. Los principios del gobierno representativo. Madrid: Alianza.

MASSETTI, A. 2009. La década piquetera (1995-2005): acción colectiva y protesta social de los movimientos territoriales urbanos. Buenos Aires: Nueva Trilce.

McADAM, D. et al. 1999. Movimientos sociales: perspectivas comparadas oportunidades políticas, estructuras de movilización y marcos interpretativos culturales. Madrid: Istmo.

MELUCCI, A. 1999. Acción colectiva, vida cotidiana y democracia. México, D. F.: El colegio de México.

MENEGUELLO, R. 1989. PT: a formação de um partido (1979-1982). Rio de Janeiro: Paz e Terra.

OFFE, C. 1992. La gestión política. Madrid: Centro de Publicaciones del Ministerio de Trabajo y Seguridad Social.

OLIVEIRA, A. U. 2009. "A política de reforma agrária no Brasil”. In: SYDOW, E.; MENDONÇA, M. L. (orgs.). Direitos humanos no Brasil 2009. São Paulo: Rede Social de Justiça e Direitos Humanos, pp.27-36.

OLIVEIRA, F. M. C. 2003. "The duckbilled platypus". New Left Review, n.24, pp.40-57.

OLSON, M. 1992. La lógica de la acción colectiva. México, D. F.: Limusa.

RADERMACHER, R.; MELLEIRO, W. 2007. "El sindicalismo bajo el gobierno de Lula”. Nueva Sociedad, n.211, pp.124-143.

RIETHOF, M. 2004. "Changing strategies of the Brazilian labor movement: from opposition to participation”. Latin American Perspectives, v.31, n.6, pp.31-47. 
RODRIGUES, L. M. 2002. Partidos, ideologia e composição social: um estudo das bancadas partidárias na Câmara dos Deputados. São Paulo: Edusp. . 2004. "Lula y los cambios en la clase política brasileña". In: RODRIGUES, L. M.; SADEK, M. A. El Brasil de Lula: diputados y magistrados. Buenos Aires: La Crujía/PNUD/ITDT.

SOARES, G. 2005. O PT e a CUT nos anos 90: encontros e desencontros de duas trajetória. Brasília: Fortium.

SVAMPA, M. 2005. La sociedad excluyente: la Argentina bajo el signo del neoliberalismo. Buenos Aires: Taurus.

TARROW, S. 1999. "Estado y oportunidades: la estructuración política de los movimientos sociales”. In: McADAM, D. et al. 1999. Movimientos sociales: perspectivas comparadas - oportunidades políticas, estructuras de movilización y marcos interpretativos culturales. Madrid: Istmo, pp.71-99.

2004. El poder en movimiento: los movimientos sociales, la acción colectiva y la política. Madrid: Alianza.

TILLY, C. 2000. “Acción colectiva”. Apuntes de Investigación del CECyP, ano 4, n.6, pp.9-32.

TOURAINE, A. 1987. El regreso del actor. Buenos Aires: Eudeba. 1995. La producción de la sociedad. México, D. F.: Unam-Ifal.

ZUAZO, M. 2010. “¿Los movimientos sociales en el poder? El gobierno del MAS en Bolivia”. Nueva Sociedad, n.227, pp.120-135. 


\section{DE LA COLONIZACIÓN DE LA SOCIEDAD CIVIL A LAS TENSIONES ENTRE PARTIDOS EN EL GOBIERNO Y MOVIMIENTOS SOCIALES}

\section{ESTEBAN IGLESIAS}

Resumo: Este trabajo se propone revisar una hipótesis planteada en la perspectiva de los movimientos sociales en el marco de la problemática de la acción colectiva. La misma considera que los puntos de contacto entre iniciativas del Estado y movimientos sociales se pueden traducir en instancias de cooptación, burocratización o colonización de esos movimientos. El argumento principal que se desarrolla plantea que el problema político principal de los movimientos sociales en América Latina actual radica en el tipo de relación que establecen con el partido que asumió el gobierno. Este corrimiento se produce en un contexto político en el que las iniciativas estatales a comienzos del tercer milenio se están orientando a reparar situaciones sociales precedentes, que se expresaron como problemas de desintegración social y política.

Palavras-chave: Partidos Políticos, Movimientos Sociales, Estado, Burocratización.

\section{FROM THE COLONIZATION OF THE CIVIL SOCIETY TO THE TENSIONS AMONG PARTIES IN GOVERNMENT AND SOCIAL MOVEMENTS}

Abstract: The present paper aims to review a hypothesis from the social movements' perspective within the frame of collective action problematic. This hypothesis considers that the contact between state's initiatives and 
social movements could be translated in cooptation, bureaucratization or colonization of social movements. The central argument maintains that the principal problem of social movements in current Latin America lies in the type of relation between social movements and the political party in government. This shift is produced in a political context in which state's initiatives at the beginning of the third millennium are being oriented to repair precedent social situations, expressed as political and social disintegration problems.

Keywords: Political Parties, Social Movements, State, Bureaucratization

Recebido: 01/08/2012 Aprovado: 29/08/2012 